Z Herz-Thorax- Gefäßchir 2008 · 22:103-109 DOI 10.1007/s00398-008-0625-5

Online publiziert: 26. März 2008

(c) Springer Medizin Verlag 2008
M. Richter $\cdot$ P. Macchiarini $\cdot$ W. Harringer

Klinik für Herz-, Thorax- und Gefäßchirurgie, Klinikum Braunschweig, BRD

\title{
Allgemeine Aspekte der chirurgischen Therapie des nichtkleinzelligen Bronchialkarzinoms
}

\section{Einleitung}

Die radikale Resektion (Ro-Resektion) ist die einzige potenziell kurative Therapieoption beim nichtkleinzelligem Bronchialkarzinom (non small cell lung cancer $=$ NSCLC). Allerdings können nur 20-30 \% aller Patienten einer Operation unter kurativer Indikation zugeführt werden. Bei der überwiegenden Zahl der Patienten liegt aufgrund der späten Diagnosestellung bereits eine Fernmetastasierung vor bzw. findet sich eine funktionelle oder allgemeine Inoperabilität.

Die Therapie des NSCLC sollte stadiengerecht nach der TNM(Tumor Nodes Metastasen)-Klassifikation (• Tabelle 1) erfolgen und in interdisziplinär besetzten Tumorkonferenzen individuell für jeden einzelnen Patienten beschlossen werden. Nach Abschluss der Diagnostik ist eine TNM-Stadienzuordnung mit Lokalisation und Ausdehnung des Primärtumors, der mediastinale Lymphknotenstatus sowie eine Aussage zum Vorliegen von Fernmetastasen zu fordern.

Hierbei ist die exakte Abklärung der Lymphknotenstationen zwingend erforderlich. Ein entsprechender Algorithmus wurde in der letzten Ausgabe dieser Zeitschrift präsentiert [1].

Ziel dieses Artikels ist es, die chirurgischen Überlegungen im Rahmen eines interdisziplinären Behandlungskonzeptes darzulegen. Eine detaillierte Beschreibung der Operationstechniken würde den Rahmen des Beitrags sprengen und bleibt entsprechenden Operationslehren sowie der Weiterbildung im Operationssaal vorbehalten.

\section{Invasives chirurgisches Staging}

Die invasive Abklärung erfolgt bei zervikalen oder supraklavikulären Lymphknoten durch offene Exstirpation oder Biopsie. Wird hier ein Malignitätsnachweis geführt, liegt stets ein $\mathrm{N}_{3}$-Status vor, der ein kuratives chirurgisches Therapiekonzept ausschließt. Obwohl die PET-CT-Untersuchung eine wertvolle Ergänzung des Staging beim NSCLC darstellt, sollten nach unserer Meinung positive mediastinale Lymphknoten einer weiteren histologischen oder zytologischen Sicherung zugeführt werden. Hierbei ist die Mediastinoskopie als Goldstandard des mediastinalen Staging anzusehen. Sie wird heute bevorzugt als Videomediastinoskopie durchgeführt und lässt eine sichere Beurteilung der Lymphknotenstationen 1, 2, (3), 4, $7 \mathrm{zu}(\bullet$ Abb. 1). Die Rate falsch-negativer Biopsiebefunde wird mit ca. $10 \%$ angegeben, der Anteil falsch-positiver Befunde ist vernachlässigbar. Die Beurteilung schlecht erreichbarer Lymphknotenstationen $(5,6$ links, 8, 9, 10 beidseits) kann durch eine anteriore Mediastinoto- mie bzw. moderner durch eine Videothorakoskopie erfolgen. Diese Techniken können auch zur Abklärung einer fraglichen Tumorinvasion in benachbarte Strukturen eingesetzt werden und so die Rate der explorativen Thorakotomien senken.

\section{Pulmonale Funktionsdiagnostik}

Angesichts der schlechten Prognose von Patienten mit nicht reseziertem NSCLC sollte ungeachtet reduzierter kardiopulmonaler Reserven die Lungenresektion

Tabelle 1

Stadieneinteilung nach TNM-

Klassifikation

\begin{tabular}{|llll}
\hline Stadium 0 & TIS & N0 & M0 \\
\hline Stadium IA & T1 & N0 & M0 \\
Stadium IB & T2 & N0 & M0 \\
\hline & & & \\
\hline Stadium IIA & T1 & N1 & M0 \\
Stadium IIB & T2 & N1 & M0 \\
& T3 & N0 & M0 \\
\hline Stadium IIIA & T1 & N2 & M0 \\
& T2 & N2 & M0 \\
& T3 & N1, N2 & M0 \\
\hline Stadium IIIB & jedes T & N3 & M0 \\
& T4 & jedes N & M0 \\
\hline Stadium IV & jedes T & jedes N & M1 \\
\hline
\end{tabular}




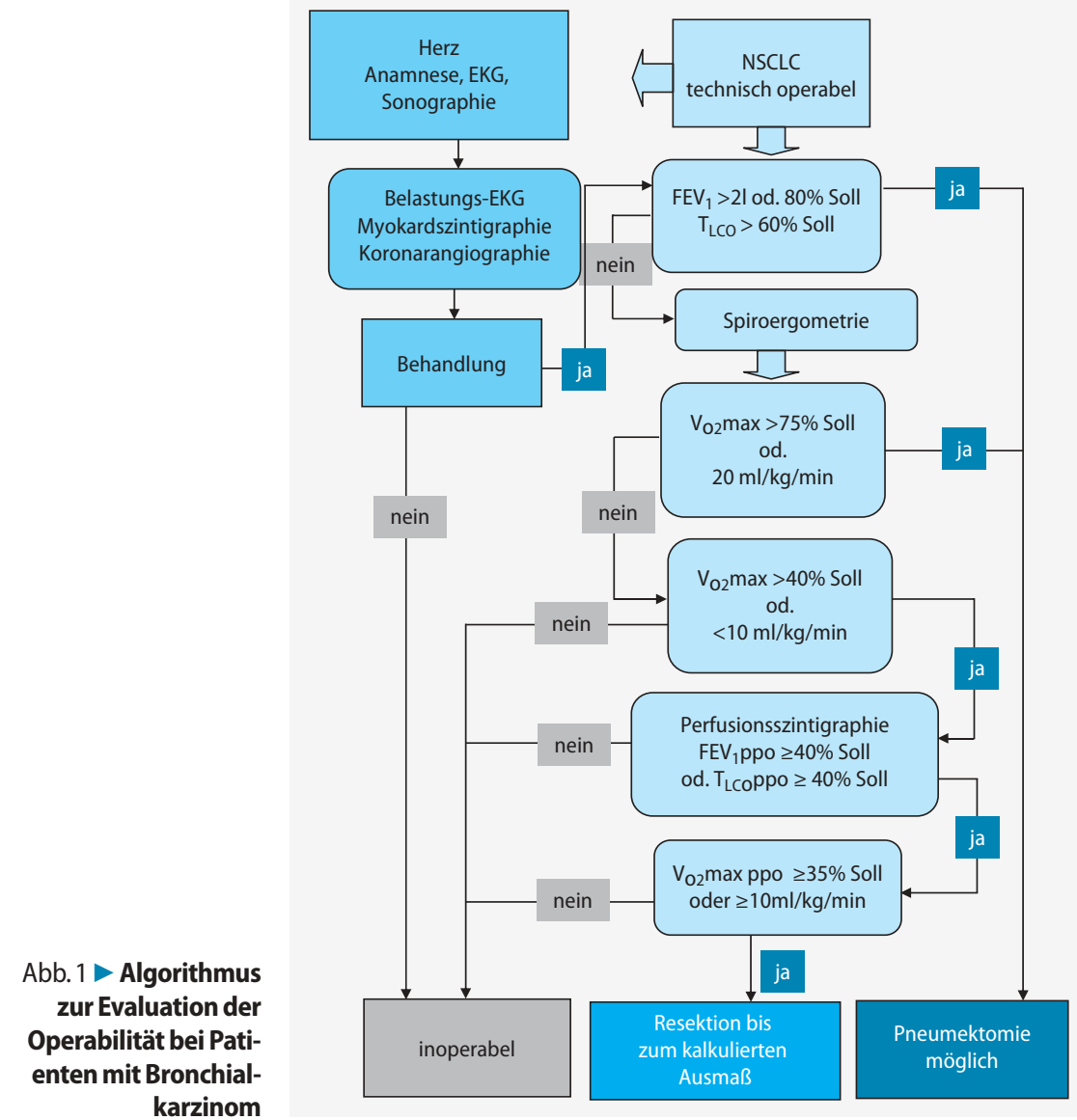

Zur Reduktion postoperativer respiratorischer Komplikationen trägt auch eine klar organisierte, meist interdisziplinär durchgeführte postoperative Schmerztherapie bei. Eine effektive Analgesie nach Thorakotomie erlaubt frühzeitige Krankengymnastik und Mobilisation sowie eine intensive Atemtherapie mit Reduktion von Sekretretention und Atelektasenbildung.

\section{Operative Behandlung}

Präoperativ beurteilt der Chirurg die Lage des Tumors und eventuelle Infiltrationen in dessen Umgebung, um die lokale Resektabilität zu evaluieren. Hierbei ist eine Überschreitung des Lungenlappenspaltes zu den angrenzenden Lungenlappen ebenso von Bedeutung wie der Abstand der Tumorgrenze zu Hauptbronchus oder Hauptkarina. Weiterhin sind Infiltrationen organüberschreitend in Thoraxwand, Zwerchfell sowie mediastinale Organe (Ösophagus, Perikard, Herz und große Gefäße) zu beachten. Nach Ergebnissen einer Heidelberger Studie zeigen

angestrebt werden. Ein entsprechender Algorithmus wird in der $\bullet$ Abb. 2 dargestellt. Er soll die präoperative Risikostratifizierung erleichtern, bedarf jedoch im Einzelfall auch einer kritischen Wertung der erhobenen Befunde und einer Abwägung derselben gegenüber einer möglichen Tumorheilung. Die Abschätzung der postoperativ zu erwartenden Lungenfunktion (forced vital capacity = FEV1-ppo, Kohlenmonoxidtransferfaktor $=$ TLCO-ppo) ist möglich, indem die Anzahl der mutmaßlich zu resezierenden Segmente gezählt und entsprechend der Formel:

$$
\begin{gathered}
\text { Wert-ppo }=\text { Wert-präop } \\
\times[(19-\text { resezierte Segmente }) / 19]
\end{gathered}
$$

die postoperativ zu erwartende Lungenfunktion berechnet wird.

Eine funktionelle Inoperabilität ist gegeben, wenn Perfusionsszintigraphie und errechnete postoperative Lungenfunktion Werte unter $40 \%$ der Norm ergeben sowie die Ergospirometrie für die $\mathrm{VO}_{2}$ max Werte unter $40 \%$ der Norm bzw. unter 10ml/ $\mathrm{kg} / \mathrm{min}$ erbringen bzw. der errechnete postoperative Wert unter $35 \%$ respektive $10 \mathrm{ml} / \mathrm{kg} / \mathrm{min}$ liegt.

\section{Tabelle 2}

\section{Geplante Modifikationen der TNM-Klassifikation}

\section{Derzeitige T-Klassifikation \\ TX positive Zytologie \\ T1 Tumor bis $\max .3 \mathrm{~cm}$ \\ T2 Tumor $>3 \mathrm{~cm}$ \\ Abstand Hauptkarina $>2 \mathrm{~cm}$ \\ partielle Atelektase \\ Invasion Pleura visceralis \\ Pericard, mediastinale Pleura, Haupt- \\ bronchus $<2 \mathrm{~cm}$, totale Atelektase \\ T4 Infiltration Ösophagus, Herz, große Gefäße, \\ Karina, Trachea \\ Satellitenherd im selben Lappen \\ maligner Pleuraerguss}

T3 Infiltration Thoraxwand, Zwerchfell, $\quad$ T3 Tumor $>7 \mathrm{~cm}$ (bisher T2)

Derzeitige N-Klassifikation

No keine Lympknotenmetastasen

N1 ipsilaterale hiläre und peribronchiale Lymphknotenmetastasen

N2 ipsilaterale mediastinale und infrakarinale Lymphknotenmetastasen

N3 kontralaterale mediastinale und hiläre Lymphknotenmetastasen ipsi- und kontralaterale Scalenus- und supraklavikuläre Lymphknotenmetastasen 
nur $63 \%$ von über 2000 operierten Patienten eine Übereinstimmung des prätherapeutischen T-Status (cT) mit dem postoperativen pathologischen T-Status (pT). Eine Unterschätzung der Ausdehnung des Primärtumors wurde in $15 \%$ der Fälle beobachtet, $22 \%$ wurden überschätzt. Als besonders problematisch stellt sich die $\mathrm{T}_{3}$ Situation dar. Bei Infiltration in Thoraxwand, Zwerchfell und mediastinale Pleura kam es in nur $35 \%$ der Patienten zu einer Übereinstimmung der Beurteilung prä- und postoperativer Befunde [2].

Nach der UICC (Union internationale contre le cancer) erfolgt derzeit die T-Stadienzuordnung entsprechend der TNMKlassifikation in einer Version aus dem Jahr 2002. Es ist jedoch vorgesehen, die TNM-Klassifikation hinsichtlich des Primärtumors zu erweitern. Auf der World Conference of Lung Cancer in Seoul im September 2007 wurde folgende geplante Neudefinition vorgestellt (• Tabelle 2 ).

In der Novellierung der TNM-Klassifikation wird eventuell eine Subklassifizierung der N2-Lymphknoten in N2a (solitäre Lymphknotenmetastasierung) und N2b (multiple Lymphknotenmetastasen) Berücksichtigung finden. Derzeit liegen hierfür jedoch nur begrenzte Daten vor. Ergänzend soll zudem die bisherige Klassifizierung der Fernmetastasierung erweitert werden. Hier ist eine Differenzierung in Mia (kontralateraler Lymphknotenbefall oder Pleurainfiltration) und $\mathrm{Mib}$ (Fernmetastasierung außerhalb von Lunge und Pleura) vorgesehen.

Die Stadienzuordnung nach der aktuellen TNM-Klassifikation (2002) ist in - Tabelle 1 dargestellt. Im Folgenden wird die chirurgische Vorgehensweise in den einzelnen Stadien besprochen.

\section{Stadium I}

Stadium I umfasst alle lokal begrenzten Tumore ohne lymphogene oder hämatogene Metastasierung. Hierbei wird ein Mindestabstand des Tumors zur Karina von $2 \mathrm{~cm}$ gefordert. Die Tumore mit einem maximalen Durchmesser bis zu $3 \mathrm{~cm}$ werden dem Stadium IA zugeordnet. Ist der Tumor größer als $3 \mathrm{~cm}$ und hat eine Ausdehnung bis maximal zur Pleura visceralis bzw. liegt eine tumorassoziierte Teilate-

Z Herz-Thorax- Gefäßchir 2008 · 22:103-109

DOI 10.1007/s00398-008-0625-5

(c) Springer Medizin Verlag 2008

\section{Richter · P. Macchiarini - W. Harringer}

\section{Allgemeine Aspekte der chirurgischen Therapie des nichtkleinzelligen Bronchialkarzinoms}

\section{Zusammenfassung}

Das Bronchialkarzinom ist eine der häufigsten Krebserkrankungen in den Industrieländern, vorrangig bei Männern, in zunehmendem Maße jedoch auch bei Frauen. Die einzig kurative Therapie besteht in der kompletten chirurgischen Resektion des Tumors. Hierbei sind die präzise präoperative Diagnostik mit möglichst exaktem Tumorstaging sowie die interdisziplinäre Diskussion und Festlegung der weiteren individuellen Therapie essenziell für ein modernes Therapiekonzept. Die operative Therapie des Bronchialkarzinoms ist heute stadienabhängig häufig in adjuvante und neoadjuvante Thera- pieschemata eingebunden und bedarf einer regen Kommunikation mit Pneumologen, Onkologen und Strahlentherapeuten. Chirurgische Überlegungen zur Therapie der einzelnen Tumorstadien werden präsentiert und die daraus resultierenden Operationsindikationen beschrieben. Darüber hinaus erläutert der Beitrag sowohl die invasiven Stagingtechniken als auch die präoperative Evaluation der Lungenfunktion.

\section{Schlüsselwörter}

Bronchialkarzinom · Chirurgie - Therapie .

Stadien

\section{General aspects of the surgical resection of lung cancer}

\section{Abstract}

Lung cancer is one of the most common cancers in industrialized nations, dominating in men but also increasing continuously in women. Complete surgical resection of the tumor remains the only curative therapy. A surgical approach requires precise preoperative diagnostic evaluation and staging of the carcinoma as well as the interdisciplinary discussion to determine the individual therapeutic treatment. Surgical therapy of lung cancer is stage dependent and often part of a multimodal approach including adjuvant or neoadju- vant radio- and/or chemotherapy. Close communication with associated specialties is mandatory. Surgical considerations for therapeutic strategies in different lung cancer stages are presented and indications for tumor resections are described. Furthermore, invasive staging techniques are discussed and preoperative evaluation of pulmonary function and its implications on extent of surgical resection are explained.

\section{Keywords}

lung cancer · therapy · surgery · staging 


\section{Weiterbildungsakademie}

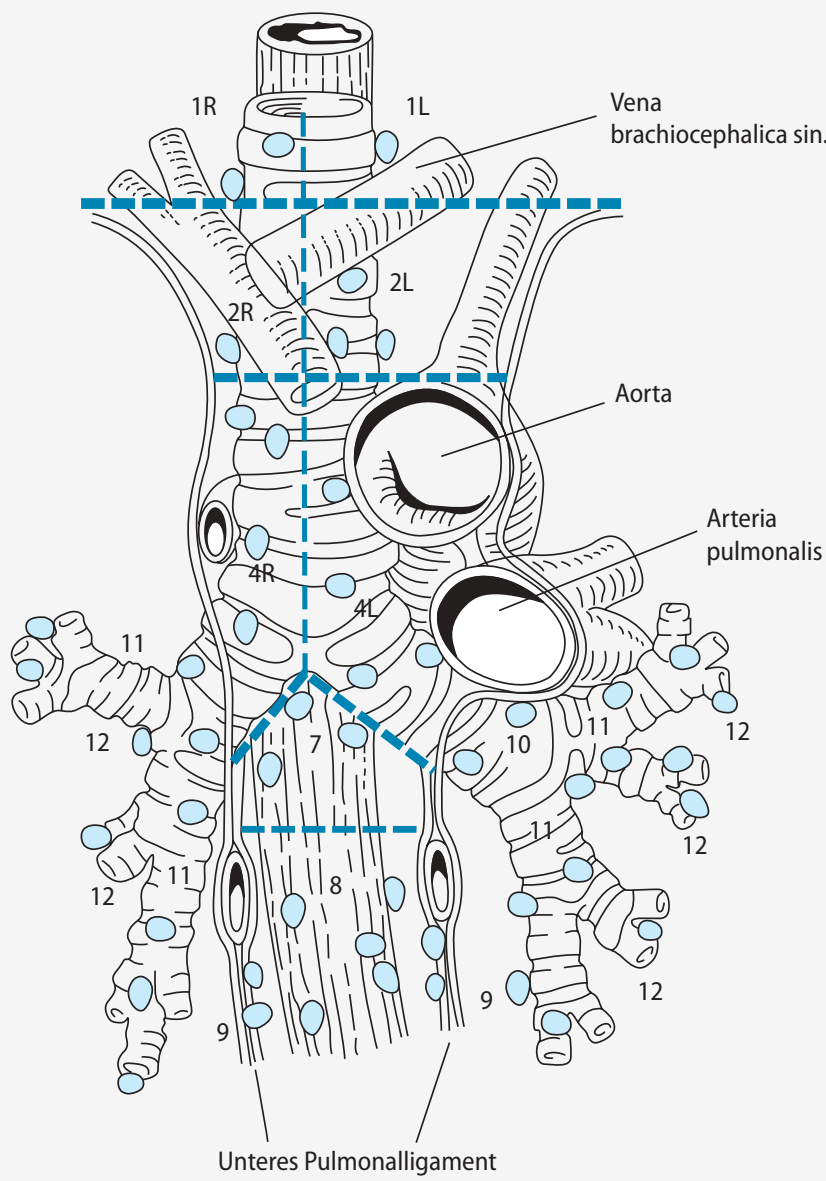

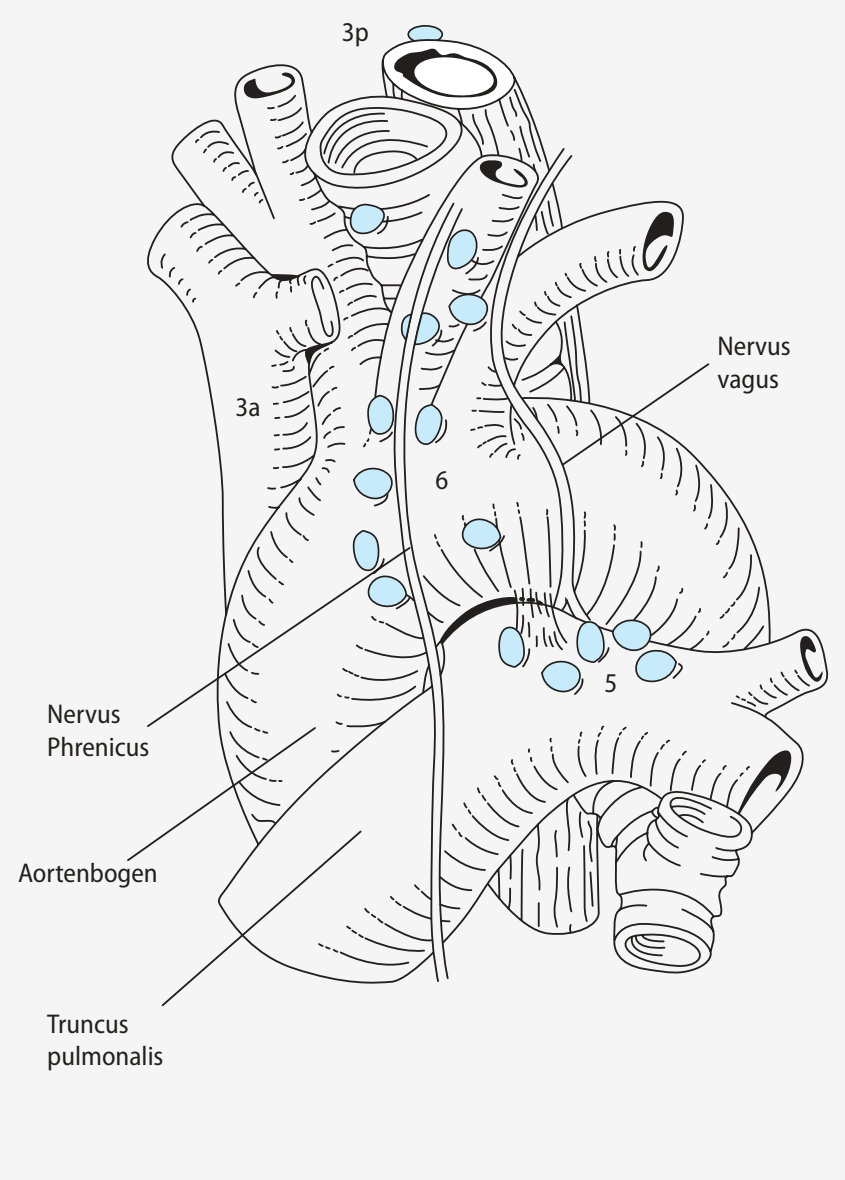

b

Abb. $2 \Delta$ Nomenklatur der Lymphknotenstationen (modifiziert nach Mountain et al. [6])

lektase vor, erfolgt eine Zuordnung in das Stadium IB.

Unter Berücksichtigung der funktionellen Operabilität und der Komorbiditäten ist in den Stadien IA und IB eine operative Resektion als Therapie etabliert. Die Standardresektion ist eine Lobektomie. Die Resektion folgt den Prinzipien der onkologischen Chirurgie mit einer kompletten En-bloc-Resektion des Tumors, ohne Tumoreröffnung mit der einhergehenden Möglichkeit der Tumorzellverschleppung. Weiterhin sollten alle Resektionsränder markiert und im intraoperativen Schnellschnittverfahren auf Tumorfreiheit histologisch untersucht werden. Sollte ein Resektionsbereich nicht tumorfrei sein, ist bei technischer Möglichkeit eine Nachresektion erforderlich.

Nach Lobektomie wird der Hemithorax von der belassenen Restlunge ausgefüllt. Der Funktionsverlust beträgt ca. 10 \% der präoperativen Einsekundenkapazität. Die Krankenhausletalität liegt bei $2 \%$ [2].
Die Lobektomie wird heute vornehmlich über eine Thorakotomie durchgeführt. Standardzugangswege zum Hemithorax sind die anterolaterale und posterolaterale Thorakotomie.

Videothorakoskopische Lobektomien unterliegen ebenfalls denselben onkologischen Kriterien wie eine offene Resektion und haben die gleichen Ergebnisse hinsichtlich der lokalen Tumorkontrolle $[3,11]$. In der videothorakoskopischen onkologischen Thoraxchirurgie ist durch eine Kombination von VATS(Video-assisted thoracic surgery)-Lobektomie und mediastinaler Lymphadenektomie via VAMLA (Video-assisted mediastinal lymph adenectomy) eine Einhaltung der Prinzipien onkologischer Chirurgie möglich. Derzeit liegen für dieses kombinerte Vorgehen noch keine Langzeitergebnisse vor.

Bei T1-Tumorerkrankungen sind sublobuläre Resektionen als anatomische Segmentresektion oder atypische Resekti- onen möglich. Diese Verfahren sollten im Rahmen von interdisziplinären Tumorkonferenzen als Einzelfallentscheidungen zum Einsatz kommen. Hierfür sind Patienten mit einer deutlich eingeschränkten Lungenfunktion, hohem Alter und/oder einer erheblichen Komorbidität qualifiziert. El-Sherif konnte zeigen, dass bei sublobulären Resektionen die lokale Tumorkontrolle mit 7,2\% gegenüber der Lobektomie mit 4,2 \% schlechter abschnei$\operatorname{det}$ [4].

T2-Tumore mit einer maximalen Ausbreitung bis zur Pleura visceralis und einem Mindestabstand zur Hauptkarina von $2 \mathrm{~cm}$ erfordern zuweilen eine Erweiterung für eine Ro-Resektion. Folgende Resektionsverfahren stehen hierfür zur Verfügung: Bilobektomie, Lobektomie oder Bilobektomie als Manschettenresektion (bronchoplastische und/oder angioplastische Erweiterung) und Pneumonektomie bzw. Manschettenpneumektomie (- Abb. 3a und b). 

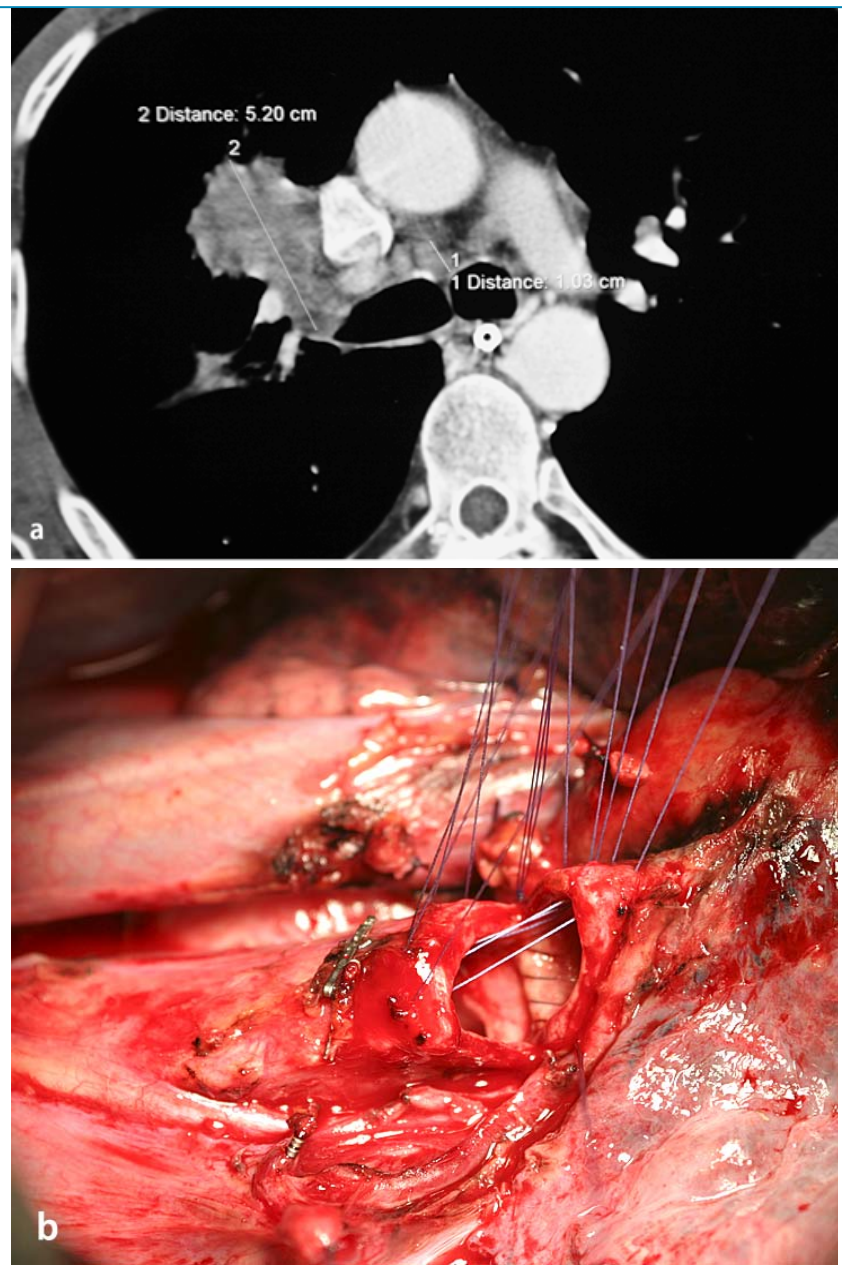

Abb. $3<$ a. Bronchialkarzinom am Oberlappenabgang rechts (CT-Untersuchung) b. Oberlappenresektion rechts als bronchiale Manschettenresektion. Der Bronchus intermedius wird an den rechten Hauptbronchus anastomosiert (Hinterwand mit fortlaufender Naht, Vorderwand mit Einzelknopfnähten; jeweils Vicryl 4-0)

Allen Standard- sowie erweiterten Resektionen folgt eine mediastinale Lymphadenektomie. Hierfür ist bislang bezüglich Nomenklatur sowie operativer Technik kein standardisiertes Vorgehen zu erkennen. Notwendige Voraussetzung für eine interdisziplinäre Therapieplanung ist jedoch eine einheitliche Nomenklatur zumindest innerhalb eines Tumorzentrums.

In der Nomenklatur der pulmonalen, hilären und mediastinalen Lymphknoten sind zweierlei Klassifikationen weit verbreitet. Naruke veröffentlichte bereits 1978 ein Schema, welches auch heute noch Bestand hat [5]. Das wohl in der Regel zur Anwendung kommende Schema stammt von Mountain aus dem Jahr 1997 [6]. In beiden Schemata werden die Lymphknotenstationen exakt beschriebenen, anatomischen Kompartimenten numerisch zugeordnet (• Abb. 1). Von klinischer Bedeutung ist die Zuordnung dieser Lymphknotenstationen zu pulmonalen und mediastinalen Lymphknotenstationen. $\mathrm{Zu}$ den pulmonalen Lymphknoten zählen die intrapulmonalen, bronchopul- monalen und die hilären Noduli. Die mediastinalen Lympknoten teilen sich auf in anteriore, tracheobronchiale, paratracheale und posterior-inferiore Lymphknotengruppen [7]. Von praktischer Bedeutung für den Chirurgen ist, dass alle pulmonalen Lymphknoten von der Pleura visceralis überzogen sind. Erfahrungsgemäß ist der Verlauf der Pleura visceralis im Lungenhilusbereich jedoch häufig nicht eindeutig zu bestimmen, weshalb die Klassifikation der dort aufgefundenen Lymphknoten zu Schwierigkeiten führen kann. Alle Lymphknoten innerhalb der Pleura visceralis auf der ipsilateralen Seite des Tumors entsprechen N1 nach TNM-Klassifikation.

Sämtliche außerhalb der Pleura visceralis und intrathorakal gelegene Lymphknoten definieren sich als mediastinale Noduli. Ipsilaterale mediastinale Lymphknoten sind als $\mathrm{N}_{2}$ und kontralaterale mediastinale Lymphknoten als $\mathrm{N}_{3}$ nach TNM klassifiziert.

Das operative Vorgehen der Lymphadenektomie bei onkologischen lungenre- sezierenden Eingriffen ist ebenso wie die Nomenklatur vielfältig. Das Spektrum reicht hier vom Sampling bis zur radikalen beidseitigen En-Bloc-Resektion der Lymphknotenkompartimente.

Beim Sampling beschränkt sich der Operateur auf die Dissektion makroskopisch pathologisch sowie palpatorisch veränderter Lymphknoten. Diese Methode ist stark von dem jeweiligen Operateur abhängig.

Unser Vorgehen bei der mediastinalen Lymphknotendissektion orientiert sich an der Veröffentlichung von Passlick und Izbicki [7]. Hierbei wird routinemäßig eine systematische mediastinale Lymphknotendissektion mit En-Bloc-Resektion der mediastinalen Lymphknotenkompartimente durchgeführt. Bei rechtsseitigen Resektionen werden das paratracheale, infrakarinale sowie das inferiore Kompartiment entfernt. Linksseitig werden das subaortale, infrakarinale und inferiore Kompartiment disseziiert. Eine Erweiterung auf das paratracheale Kompartiment ist anzustreben (• Abb. 1).

Patienten im Stadium I haben nach Raz im natürlichen Krankheitsverlauf eine 5Jahres-Überlebenswahrscheinlichkeit von $0,42 \%$ [8]. Es wurden hier 1432 Patienten im Stadium I verfolgt (Gesamtzahl der Patienten im Stadium I = 19702), welche sich nach Diagnose der Tumorerkrankung keiner Therapie unterzogen. Berücksichtigung fand hier die natürliche Sterbequote, welche bei Patienten ohne Therapie des Bronchialkarzinoms aufgrund multipler Komorbiditäten deutlich erhöht ist.

Nach kurativer operativer Therapie im Stadium I ist eine 5-Jahres-Überlebenswahrscheinlichkeit von ca. $70 \%$ für das Stadium IA und von ca. 60\% im Stadium IB zu erwarten. Die Rezidivrate beläuft sich im Stadium IA auf $10 \%$ für lokale und $15 \%$ für systemische Rezidive. Im Stadium IB beträgt die lokale Rezidivrate ebenfalls $10 \%$; die systematische Rezidivrate liegt bei $30 \%[9,15]$.

Mehrere Studien befassten sich mit der Fragestellung einer platinbasierten adjuvanten Chemotherapie in den Stadien IA und IB. Auf dem ASCO (American Society of Clinical Oncology) 2006 wurde die Metaanalyse LACE (lung adjuvant Cisplatin evaluation) mit einem Datenpool der 5 großen Studien (ALPI, ANITA, BLT, IALT, 
JBR10) veröffentlicht [10]. Hierbei zeigt sich für das Stadium IA kein Überlebensvorteil durch adjuvante Chemotherapie nach kurativer Operation. Im Stadium IB ist die Datenlage nicht eindeutig. Die Studien JBR1o und ANITA zeigen einen nicht signifikanten Überlebensvorteil von $76 \%$ versus $69 \%$ bzw. $62 \%$ versus $63 \%$. In den Guidelines des National Comprehensive Cancer Network 2007 wird eine Empfehlung Kategorie IIB gegeben, basierend auf einer Low-level-Evidenz einschließlich klinischer Erfahrungen [12]. Bei der Indikationsstellung zur adjuvanten Chemotherapie sind hier der Allgemeinzustand des Patienten, einschließlich der Komorbiditäten hinsichtlich einer platinbasierten Chemotherapie zu berücksichtigen. Die klinische Erfahrung zeigt, dass bei pneumektomierten Patienten chemotherapiebedingte Nebenwirkungen häufiger auftreten und diese Patienten die Chemotherapie deshalb toxizitätsbedingt vorzeitig abbrechen.

Eine generelle Empfehlung für eine adjuvante Chemotherapie im Stadium IB kann nicht gegeben werden.

\section{Stadium II}

Das Tumorstadium II ist heterogen. Es umfasst die Stadien IIA $\left(\mathrm{T}_{1} \mathrm{~N}_{1}\right)$ sowie IIB ( $\mathrm{T}_{2} \mathrm{~N}_{1}$ und $\mathrm{T}_{3} \mathrm{No}$ ).

Hinsichtlich der chirurgischen Therapie ergeben sich keine Änderungen zur Therapie des Bronchialkarzinoms im Stadium I. Es erfolgt eine Tumorresektion als Lobektomie, Bilobektomie, bronchoplastische und/oder angioplastische Manschettenresektion oder Pneumonektomie (- Abb. 3a und b).

Die Datenlage aus den adjuvanten Chemotherapiestudien bezüglich der pN1-Situation ist gegenüber Stadium IB (pNo) eindeutig. Die bereits oben genannten Studien zeigen einen signifikanten Überlebensvorteil: JBR $1062 \%$ versus $42 \%$ und ANITA $52 \%$ versus $39 \%$. Es gibt eine Empfehlung Kategorie I, basierend auf einer High-level-Evidenz [12]. Die Chemotherapie sollte bis zum 42. postoperativen Tag beginnen.

Die Heterogenität des Stadiums II ist durch $\mathrm{T}_{3}$ No bedingt. Die Prognose der Tumorerkrankung verschlechtert sich in Abhängigkeit vom Ort der organüber- schreitenden Infiltration. Hierbei ist eine Infiltration der Thoraxwand prognostisch günstiger als die Infiltration des Hauptbronchus oder der mediastinalen Pleura und des Zwerchfells [13].

Ziel der operativen Therapie ist auch bei Organüberschreitung eine lokale Tumorkontrolle durch eine Ro-Resektion. Dies wird durch eine En-Bloc-Resektion des tumortragenden Lungenlappens einschließlich Thoraxwandteilresektion erreicht. Bei kleineren Defekten der Thoraxwand sind ein primärer Wundverschluss oder eine Jalousieplastik nach Heller möglich. Sollte bei ausgedehnten Thoraxwandresektionen eine Rekonstruktion erforderlich werden, stehen verschiedene operative Methoden zur Verfügung. Für die Stabilisierung der Thoraxwand kommen Polypropylen Mesh, Polygalactin Mesh sowie Polytetrafluorethen Mesh zum Einsatz. Bei Notwendigkeit einer soliden Stabilisierung werden die Netze im Sinne der Sandwich-Technik mit Methylmethacrylat kombiniert. Meist ist eine Weichteildeckung ohne plastische Erweiterung möglich. Erfordert der Defekt nach der Resektion eine plastische Deckung, kann dies durch Muskellappenplastiken des Musculus pectoralis major oder Musculus latissimus dorsi respektive bei großen Defekten durch myokutane Lappenplastiken des Musculus latissimus dorsi oder freie Lappenplastiken erfolgen.

Bei zentraler Infiltration des Tumors Richtung Hauptbronchus sind bronchoplastische und/oder angioplastische Resektionen mit Erhalt von Lungenlappen einer Pneumektomie vorzuziehen.

Die 5-Jahres-Überlebenswahrscheinlichkeit im gesamten Stadium II liegt bei ca. $40 \%$. In den Substadien belaufen sich die unterschiedlich hohen 5-Jahres-Überlebenswahrscheinlichkeiten für $\mathrm{T}_{1} \mathrm{~N}_{1}$ auf $54 \%, \mathrm{~T}_{2} \mathrm{~N}_{1}$ auf $40 \%$ und $\mathrm{T}_{3} \mathrm{No}$ auf $38 \%$. Hierbei ist die mediastinale Tumorinfiltration prognostisch als ungünstig anzusehen: Literaturangaben variieren zwischen $7 \%$ und $25 \%$ [14].

\section{Stadium III}

Stadium IIIA umfasst Bronchialkarzinome $\mathrm{T}_{1}-2 \mathrm{~N}_{2}$ sowie $\mathrm{T}_{3} \mathrm{~N}_{1}-2$.

Der Primärtumor ist hinsichtlich seiner pathologischen Ausdehnung maximal
T3 und unterliegt damit den onkologischchirurgischen Resektionsverfahren wie in den Stadien I und II.

Tumore im Stadium IIIA als $\mathrm{T}_{3} \mathrm{~N}_{1}$ haben eine bessere Prognose als alle mediastinal metastasierten Bronchialkarzinome. Das therapeutische Vorgehen beginnt mit einer operativen En-Bloc-Tumorresektion, gefolgt von einer adjuvanten platinbasierten Chemotherapie.

Eine besondere Entität stellen hier die Pancoast-Tumore dar. Diese von der Lungenspitze ausgehenden Tumore infiltrieren den Apex des Hemithorax und bedingen ein multimodales Therapiekonzept. Einer Initialtherapie mit kombinierter Radio-/Chemotherapie folgt die operative Resektion [15].

Im Wesentlichen ist das Stadium IIIA durch die ipsilaterale mediastinale Lymphknotenmetastasierung gekennzeichnet. Nach Robinson werden folgende Subgruppen differenziert [16]:

III A1 inzidentelle Lymphknotenmetastasen N2, Nachweis in Operationsresektaten

III A2 Single-level-Metastasen N2, Nachweis intraoperativ

III A3 Single- oder Multi-level-Metastasen $\mathrm{N}_{2}$, Nachweis prätherapeutisch

III A4 Bulky- oder multi-level-Metastasen $\mathrm{N}_{2}$

Die Subgruppe IIIA1 umfasst Patienten, bei denen nach klinischem Staging Stadium I oder II diagnostiziert wurden. Mit den heute zur Verfügung stehenden Methoden sollte eine mediastinale Lymphknotenmetastasierung möglichst prätherapeutisch erfasst werden.

Im therapeutischen Konzept des Stadiums IIIA1-2 wird nach der Operation eine adjuvante Chemotherapie empfohlen. 20-30 \% der Patienten haben zum Zeitpunkt der Diagnosestellung Mikrometastasen oder isolierte Tumorzellen, 40-60 \% werden Organmetastasen entwickeln. Die Studien ANITA und IALT zeigten für diese Patientengruppe einen Überlebensvorteil von $14 \%$ in 5 Jahren $[17,18]$.

Für die Stadien IIIA3-4 liegt eine evidenzbasierte Therapieempfehlung derzeit nicht vor. Die aktuellen multimodalen Therapiekonzepte umfassen eine definitive Radio-Chemotherapie, Radio-Che- 
motherapie mit anschließender Operation sowie Induktionschemotherapie gefolgt von Operation mit oder ohne anschließende Radiotherapie.

Die Operation nach neoadjuvanter Therapie ist mit einer höheren Komplikationsrate verbunden. Hierbei ist das Komplikationsrisiko bei präoperativer RadioChemotherapie im Vergleich zur alleinigen Induktionschemotherapie deutlich erhöht. Mögliche Ursache ist eine systemische Wirkung der neoadjuvanten Therapie auf das Immunsystem mit lokalen entzündlichen Folgen. Weiterhin werden intraoperativ Gewebefibrosierungen vorgefunden, welche zu vermehrtem intraoperativen Blutverlust, längeren Operationszeiten und schwierigeren Operationsbedingungen führen. Durch die Fibrosierung sind die Präparationsebenen verschmolzen.

Besonders ist hier auf das vermehrte Auftreten von Bronchusstumpfinsuffizienzen hinzuweisen. Eine intraoperative Deckung des Bronchusstumpfes ist mittels eines Interkostalmuskellappens oder der Vena azygos (rechts) und/oder Pleura parietalis möglich. Neuere Techniken verwenden einen intrathorakalen Fettlappen als perikardialen oder phrenikoperikardialen Lappen.

Stadium IIIB ist hauptsächlich durch die kontralaterale mediastinale Lymphknotenmetastasierung gekennzeichnet. Hier hat ein chirurgisches Vorgehen keinen Stellenwert; die Patienten werden einer kombinierten Radio-Chemotherapie zugeführt.

Ist das Stadium IIIB durch T4 als Satellitenknoten im gleichen Lungenlappen definiert und konnte der Lymphknotenstatus No gesichert werden, können mit chirurgischer Resektion 5-Jahres-Überlebenswahrscheinlichkeiten wie in den Stadien IB und II erreicht werden [19]. Ebenso sind bei Infiltration von Vena cava superior oder Karina bei nodal negativen Tumoren und kompletter Resektion gute Langzeitergebnisse zu erreichen. Hierbei handelt es sich jedoch um wenige, selektionierte Patienten, die sich einem derartigen Eingriff im $\mathrm{T}_{4}$-Stadium unterziehen [20].

\section{Stadium IV}

Bei Existenz einer einzelnen Metastase (ZNS, Nebenniere) bei T1-2No-Tumoren sollten Metastase und Primärtumor reseziert und eine adjuvante Chemotherapie bzw. Ganzhirnbestrahlung angeschlossen werden. Dieses Vorgehen repräsentiert jedoch die Ausnahme, da die überwiegende Zahl von Patienten in diesem Stadium von einer chirurgischen Therapie nicht profitieren.

\section{Literatur}

1. Dickgreber NJ, Fischer S, Welte T (2008) Präoperatives Staging bei Patienten mit nichtkleinzelligem Bronchialkarzinom. Z Herz Thorax Gefäßchir 22: 56-62

2. Hoffmann H, Bülzebruck H, Dienemann H (2002) Chirurgische Therapie des nichtkleinzelligen Bronchialkarzinoms. Onkologie 8:425-433

3. McKenna RJ Jr, Houck W, Fuller CB (2006) Videoassisted thoracic surgery lobectomy: Experience with 1,100 cases. Ann Thorac Surg 81:421-426

4. El-Sherif A, Gooding WE, Santos R, Pettiford B, Ferson PF, Fernando HC, Urda SJ, Luketich JD, Landreneau RJ (2006) Outcomes of sublobar resection versus lobectomy for stage I non-small cell lung cancer: a 13-year analysis. Ann Thorac Surg 82: 408-416

5. Naruke, T; Suemasu, K; Ishikawa, S (1978) Lymph node mapping and curability at various levels of metastasis in resected lung cancer. J Thorac Cardiovasc Surg 76:832

6. Mountain CF, Dresler CM (1997) Regional lymph node classification for lung cancer staging. Chest 111:1718

7. Passlick B, Izbicki J.R. (2003) Lymphknotendissektion. In: Drings $\mathrm{P}$, Dienemann $\mathrm{H}$, Wannemacher $\mathrm{M}$ (Hrsg.) Management des Lungenkarzinoms. Springer Verlag, Berlin Heidelberg New York Hongkong Mailand Paris Tokio, 239-247

8. Raz DJ, Zell JA, Ou SH, Gandara DR, Anton-Culver $\mathrm{H}$, Jablons DM (2007) Natural history of stage I non-small cell lung cancer: implications for early detection. Chest 132:193-199

9. Pisters KM, Le Chevalier T (2005) Adjuvant chemotherapy in completely resected non-small-cell lung cancer. J Clin Oncol 23:3270-3278
10. Pignon, JP, Tribodet, H, Scagliotti, GV, et al. (2006) Lung Adjuvant Cisplatin Evaluation (LACE): A pooled analysis of five randomized clinical trials including 4,584 patients. J Clin Oncol 24:366s

11. Mun, M; Kohno,T (2007) Video-assisted thoracic surgery for clinical stage I lung cancer in octogenarians. Ann Thorac Surg 85: 406-412

12. NCCN clinical practice guidelines in oncology: non-small cell lung cancer V.I.2007: MS-1; URL: http://www.ncen.org (01.02.2007)

13. Ginsberg RJ, Martini N (2002) Non-small cell lung cancer - surgical management. In: Pearson FG, Cooper JD, Deslauriers J, Ginsberg RJ, Hiebert CA, Patterson GA, Urschel HC (eds.)Thoracic surgery. Churchill Livingstone, New York Edinburgh London Philadelphia, pp 837-859

14. Hoffmann H, Dienemann H, Passlick B (2006) Chirurgische Therapie des nichtkleinzelligen Bronchialkarzinoms Stadium I/II. Der Onkologe 12:728-736

15. Macchiarini $P$ (2004) Resection of superior sulcus karzinomas. Thorac Surg Clin 14:229-240

16. Depierre A, Milleron B, Moro-Sibilot $D$, Chevret $S$, Quoix E, Lebeau B, Braun D, Breton JL, Lemarié E, Gouva S, Paillot N, Bréchot JM, Janicot H, Lebas FX, Terrioux P, Clavier J, Foucher P, Monchâtre M, Coëtmeur D, Level MC, Leclerc P, Blanchon F, Rodier JM, Thiberville L, Villeneuve A, Westeel V, Chastang C; French Thoracic Cooperative Group (2002) Preoperative chemotherapy followed by surgery compared with primary surgery in resectable stage I (except T1N0), II, and Illa non-small-cell lung cancer. J Clin Oncol 20:247-253

17. International Adjuvant Lung Cancer Trial Collaborative Group (2004) Cisplatin-based adjuvant chemotherapy in patients with completely resected non-small-cell lung cancer. N Engl J Med 350: 351-360

18. Douillard JY, Rosell R, De Lena M, Carpagnano F, Ramlau R, Gonzáles-Larriba JL, Grodzki T, Pereira JR, Le Groumellec A, Lorusso V, Clary C, Torres AJ, Dahabreh J, Souquet PJ, Astudillo J, Fournel P, Artal-Cortes A, Jassem J, Koubkova L, His P, Riggi M, Hurteloup P (2006) Adjuvant vinorelbine plus cisplatin versus observation in patients with completely resected stage IB-IIIA non-small-cell lung cancer (Adjuvant Navelbine International Trialist Association [ANITA]): a randomised controlled trial. Lancet Oncol 7:719-727

19. Port JL, Korst RJ, Lee PC, Kansler AL, Kerem Y, Altorki NK (2007) Surgical resection for multifocal (T4) non-small cell lung cancer: is the T4 designation valid? Ann Thorac Surg 83:397-400

20. Macchiarini P, Chapelier AR, Monnet I, Vannetzel JM, Rebischung JL, Cerrina J, Parquin F, Ladurie FL, Lenot B, Dartevelle PG (1994) Extended operations after induction therapy for stage Illb (T4) non-small cell lung cancer. Ann Thorac Surg 57:966-973

\section{Korrespondierender Autor}

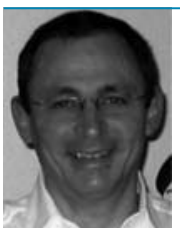

OA Dr. med. Matthias Richter

Klinik für Herz-, Thorax- und Gefäßchirurgie

Klinikum Braunschweig gGmbH

Salzdahlumer Straße 90, 38126 Braunschweig, BRD

E-Mail: htg@klinikum-braunschweig.de

OA Dr. med. Matthias Richter, Jahrgang 1959, absolvierte sein Medizinstudium in Leipzig und seine chirurgische Ausbildung in Stendal, Magdeburg und Hannover. 1991 erwarb er den Facharzt für Chirurgie, 1993 und 1999 folgten jene für Gefäßchirurgie sowie Herz- und Thoraxchirurgie. Seit 2003 ist Richter Bereichsleiter der Thoraxchirurgie in der HTG-Klinik des Städtischen Klinikums Braunschweig.

Interessenkonflikt. Der korrespondierende Autor gibt an, dass kein Interessenkonflikt besteht. 